Estado nutricional de adolescentes:

"risco de sobrepeso" e "sobrepeso" em uma escola pública do Município de São Paulo

\author{
Nutritional status of adolescents: \\ "risk of overweight" and "overweight" \\ in a public school in São Paulo
}

Renata Doratioto Albano 1

Sônia Buongermino de Souza 2

\footnotetext{
1 Universidade

Católica de Santos.

Av. Ana Costa 95, Santos,SP

11060-001, Brasil.

rdalbano@bol.com.br

2 Departamento de Nutrição,

Faculdade de Saúde Pública,

Universi dade de São Paulo.

Av. Dr. Arnaldo 715 ,

São Saulo, SP

01246-904, Brasil.

buonger@usp.br
}

Abstract Thisstudy aimed to assess the nutritional status of adolescents. A cross-sectional study was taken in a public high school in São Paulo, Brazil, with 92 students ages 11 to 17 years. The sample was performed using systematic statistical selection. Nutritional status of adolescents was assessed using body mass index (BMI), compared to WHO (1995) standards. Prevalence rates for "risk of overwei ght" and "overwei ght" were $27.9 \%$ and $4.6 \%$, respectivel y, for boys, as compared to $10.2 \%$ and $16.3 \%$ for girls. Prevalence of "risk of overweight" and "overweight" was thus high for both sexes; the results were similar in comparison to other studies. In addition, the difference in nutritional status between genders was significant $(p<0.05)$.

Key words Adolescence; Anthropometry; Body Mass Index; Nutritional Status

Resumo Com objetivo de avaliar o estado nutricional de adolescentes, foi realizado um estudo transversal em escola pública de ensino fundamental e médio no Município de São Paulo, com 92 alunos de 5ạ a 8a séries do ensi no fundamental, entre 11 e 17 anos, matriculados em 1998. A amostra foi seleci onada utilizando-se um procedimento sistemático, de acordo com a série e sexo. Com as variáveis peso e altura, calculou-se o Índice de Massa Corporal (IMC) para a idade e sexo, e os pontos de corte escolhi dos para classificar os adolescentes quanto ao estado nutricional deacordo com o percentil do IMC, foram os recomendados pela Organização Mundial de Saúde (WHO, 1995). As prevalências de "risco de sobrepeso" e "sobrepeso" foram para o sexo masculino de $27,9 \%$ e 4,6\%, respectivamente, e para o sexo femi ni no foram de $10,2 \%$ e $16,3 \%$, respectivamente. Concluiu-se que as prevalências de "risco de sobrepeso" e "sobrepeso" foram el evadas em ambos os sexos e semel hantes àquel as encontradas na literatura, havendo di ferença estati sticamente significativa no estado nutricional entre os sexos $(p<0,05)$.

Palavras-chave Adolescência; Antropometria; Índice de Massa Corporal; Estado Nutricional 
Introdução

A adolescência é uma fase caracterizada por profundas transformações somáticas, psicológicas e sociais (Saito, 1993).

Cronologicamente, a adolescência corresponde ao período de 10 a 19 anos, sendo este dividido em duas fases: fase 1-10 a 14 anos, e fase 2-15 a 19 anos. A faixa etária compreendida entre 10 e 14 anos inclui o início das mudanças puberais. O término da fase de crescimento e de desenvolvimento morfológicos ocorre no período de 15 a 19 anos (OMS, 1995).

Como o final do adolescer é um período impreciso, que prevê a consolidação da identidade, indepen dência da família, amadurecimento da sexualidade e da afetividade, autodeterminação e responsabilidade, introduziu-se o termo juventude como um prolongamento da adolescência, que coincide com a etapa póspuberal. Esta compreende o período entre 15 a 24 anos de idade (OMS, 1995; OPS, 1995).

De acordo com Ojeda \& Karin, em 1993, a adolescência implica uma série de mudanças morfológicas, funcionais e psicológicas, conduzindo a um completo dimorfismo sexual, aquisição da capacidade reprodutora e o aparecimento de novos modos de comportamento (Ojeda \& Karin, 1993, apud OPS, 1995).

Dos problemas nutricionais que acometem os adolescentes, pode-se destacar nos últimos anos o aumento do sobrepeso e obesidade. Nos Estados Unidos, a preval ência de sobrepeso tem aumentado muito, em um período curto de tempo. Em alguns subgrupos populacionais de crianças e adolescentes, aproximadamente entre 11 e $25 \%$ são consi derados com sobrepeso ou risco de sobrepeso (Troiano \& Flegal, 1998).

De acordo com dados do National Health and Nutrition Examination Survey III (NHANESIII), conduzido de 1988a 1994 pelo National Center for Health Statistcs (NCHS) pertencente ao Centers for Disease Control and Prevention (CDC), a prevalência de sobrepeso em adolescentes entre 12 e 17 anos foi $10,6 \%$. No sexo masculino, na faixa etária de 12 a 14 anos, a prevalência de sobrepeso foi de $10,7 \%$, e para a faixa etária de 15 a 17 anos, foi de 12\%; para o sexo feminino, as prevalências foram de $11,5 \%$ para a faixa etária de 12 a 14 anose $8,2 \%$ para a faixa etária de 15 a 17 anos (Troiano \& Flegal, 1998).

Segundo Kennedy \& Goldberg (1995), o aumento na prevalência de sobrepeso reflete uma população com balanço energético positivo; porém, nem os dados do National Health and Nutrition Examination (NHANESI, II e III) e nem outros dados norte-americanos indicaram aumento no consumo energético entre crian- ças e adol escentes. O consumo energético entre os anos 70 e 90 permaneceu praticamente inalterado, haven do diminuição de consumo em alguns grupos etários.

Deve ser mencionado, também, que a prática da ati vidade física vem diminuindo entre os adolescentes, nos Estados Unidos. Health et al. (1994) verificaram, em uma pesquisa com estudantes do ensino médio, que a participação dos alunos nas aulas de educação física, bem como a prática de esportes extracurricular e a participação em programas de recreação diminuíram no período de 1984 a 1990. Além disso, para Gutin e Manos, em 1993, as mudanças nos hábitos de trabalho, assistir à tel evisão e usar jogos el etrônicos por longos períodos de tempo, e outros aspectos culturais ligados ao meio ambiente, diminuem as oportunidades para a prática de atividade física (Troiano \& Flegal, 1998).

O aumento no consumo de alimentos gordurosos, com al ta densidade energética, e a diminuição na prática de exercícios físicos são os dois principais fatores, ligados ao meio ambiente, que colaboram para o aumento da prevalência da obesidade (Hill \&Trowbridge, 1998), al ém de estudos recentes também mostrarem que o acúmulo/gasto de gordura corporal é regulado geneticamente (Rosenbaum \& Leibel, 1998).

Sabe-se que existe grande probabilidade de a criança e de o adolescente obesos tornaremse adultos obesos, podendo também apresentar outras patologias associadas à obesidade, como hipertensão, dislipidemia e diabetes não insulino dependente (Dietz, 1998).

Quanto à avaliação do estado nutricional, na adol escência, a antropometria é especialmente importante porque permite monitorar a evolução das modificações do crescimento. Além disto, durante a fase de crescimento, o indivíduo pode estar sujeito tanto aos déficits nutricionais quanto aos excessos, e a antropometria é um indicador do estado nutricional e de risco para a saúde (WHO, 1995).

$\mathrm{Na}$ adolescência, além do sexo e da idade, o estágio de maturação sexual também é um fator importante na interpretação dos dados antropométricos. O ideal é utilizar um indicador para o início do estirão puberal e um indicador para verificar o término da velocidade máxima de crescimento da altura e mudanças associadas. Para o sexo masculino considera-se como característica "voz de adulto" e "órgãos genitais externos no estágio 3 de desenvolvimento"; para o sexo feminino, as características a serem consideradas são a menarca e "mamas no estágio 2 de desenvolvimento" (WHO, 1995). 
Alguns pesquisadores avaliam essas características do estágio de maturação sexual, com base em informações fornecidas pelos próprios adolescentes, com o auxílio de fotografias. Porém, não é recomendada a utilização indiscriminada da informação do próprio indivíduo, pois os estudos que utilizaram este tipo de avaliação foram feitos apenas em adolescentes brancos americanos (WHO, 1995).

As relações que levam em consi deração peso e al tura apresentam grande precisão porque essas medições oferecem baixa margem de erro, desde que as técnicas para a tomada das medidas sejam seguidas adequadamente. O Índice de Massa Corporal (IMC) é a relação mais conhecida. O cál culo é feito dividindo-se o peso, expresso em quilogramas, pela altura expressa em metros e elevada ao quadrado: IMC = peso (kg)/altura2 (metros) (Monteiro, 1998b).

Existem controvérsias na literatura em se utilizar o IMC para medir adiposidade. Para alguns autores, este índice é utilizado somente para classificar indivíduos com sobrepeso e não obesidade, pois não mede quantidade de gordura corporal (Troiano \& Flegal, 1998).

Um outro problema em relação ao IMC é o estabelecimento dos pontos de corte para avaliar sobrepeso e obesidade. Há a necessi dade de que esses pontos de corte sejam estabelecidos especificamente para cada população de acordo com suas características de desenvolvimento, por meio de estudos longitudinais (Bellizzi \& Dietz, 1999). No Brasil, não há dados sobre estudos Iongitudinais com adolescentes. Desta forma, utilizam-se os pontos de corte estabelecidos para a população americana (estudos do NCHS) e recomendada como referência pela Organização Mundial da Saúde.

Al guns estudos com crianças e adolescentes, contudo, mostraram associação entre IMC e gordura corporal como os de Pietrobelli et al. (1998), realizado na Itália, e de Daniels et al . (1997), nos Estados Unidos.

No Brasil, não há dados sobre inquéritos nacionais utilizando-se o IMC em adol escentes, mas somente dados de estudos locais/ regionais. Na Pesquisa Nacional de Saúde e Nutrição (PNSN), realizada pelo Instituto Nacional sobre Saúde e Nutrição (INAN), em 1990, foram observados déficits de $7 \mathrm{~cm}$ em altura aos 13 anos para as meninas e de $9 \mathrm{~cm}$ aos 14 anos para os meninos, em relação à população do NCHS, utilizada como referência. Essa pesquisa não utilizou o IM C para a avaliação antropométrica, mas sim os indicadores de peso/ altura, altura/idade e peso/ idade (MS, 1990).

Esses aspectos evidenciam a importância de se conhecer melhor a prevalência de "risco de sobrepeso" e "sobrepeso" em crianças e adolescentes brasileiros visando à prevenção.

Devido ao exposto, o objetivo do presente estudo foi avaliar a prevalência de "risco de sobrepeso" e "sobrepeso" em um grupo de adolescentes.

\section{$M$ ateriais e métodos}

Este estudo é do tipo transversal ou de prevalência e foi desenvolvido em escola pública, de Ensino Fundamental e Médio, do Município de São Paulo.

A população de estudo foi composta por alunos de 5 a a 8 a séries do Ensino Fundamental (10 grau), na faixa etária de 11 a 17 anos, matriculados em 1998.

O tamanho da amostra foi calculado baseando-se na precisão desejada para se estimar a proporção de adolescentes com peso e altura adequados para a idade e sexo. A fórmula utilizada para o cálculo foi:

$$
\mathrm{n}_{0}=\text { p.q.z2/ } \mathrm{d}^{2}
$$

Onde:

$\mathrm{p}=$ proporção esperada de adolescentes com peso e altura adequados;

$q=1-p$;

z = percentil da Distribuição Normal;

d =erro máximo em valor absoluto (Silva, 1998);

E considerando $p=80 \%$ e $d=5 \%$ para $u m$ intervalo de confiança de $95 \%$, tem-se:

$\mathrm{n}_{0}=0,80 \cdot 0,20 \cdot 1,962 / 0,052 \sim 246$

Para se obter a estimativa final do tamanho da amostra, o valor de $\mathrm{n}_{0}$ foi ajustado usando-se um fator de correção para populações finitas:

$$
\mathrm{n}=\mathrm{n}_{0} / 1+\mathrm{n}_{0} / \mathrm{N}
$$

Onde $\mathrm{N}=$ número total dos alunos de $5 \underline{a}$ a 8a séries matriculados em 1998

$$
n=246 / 1+246 / 251 \cong 125
$$

A amostra foi selecionada usando-se um procedimento de amostragem sistemática, com intervalo 2 (Silva, 1998), consoante a série que o aluno estava cursando e o sexo. Para a seleção da amostra, foi feita uma listagem com todos os nomes dos alunos de 5 a a 8 a séries, dispostos por série e por sexo (todos os meninos da $5 a$ série, em seguida todos os meninos da $6 \underline{a}$ série, todos os meninos da 7ạ série e todos os meninos da 8a série, sendo utilizado o mesmo procedimento para o sexo feminino).

Para caracterizar os adolescentes, foram coletadas informações quanto às características sócio-econômicas da família dos adolescentes, mediante questionário enviado para os pais e/ ou responsáveis responderem, do qual constavam os itens: 
- Renda da família: coletada em reais e transformada em categorias de salários mínimos. Estas faixas foram baseadas nas citadas no Anuário Estatístico do Brasil do IBGE - Fundação Instituto Brasileiro de Geografia e Estatística (1997). O valor do salário mínimo utilizado foi o divulgado oficialmente em maio de 1999 $\mathrm{R} \$ 136,00$ (cento e trinta e seis reais).

- Escolaridade dos pais: categorizada em: até 4ạ série, 5a a 8a séries, 1o ao 3o colegial, superior incompleto, superior completo, pós-graduação.

- Ocupação/cargo dos pais: foi descrita de acordo com a classificação utilizada na Pesquisa Nacional por Amostra de Domicílios número 05 - IBGE (1990): “Para a avaliação antropométrica,utilizaram-se as variávei s peso ealtura. Com as medidas de peso ealtura, calculouseo Índicede Massa Corporal (I MC). Com os vaIores do IMC, observaram-se os percentis correspondentes a eles, de acordo com a idadee sexo para classificar o estado nutricional dos adolescentes".

Os pontos de corte dos percentis (de acordo com o IMC) para a idade e sexo propostos pelo NCHS (WHO, 1995) são:

- Magreza ou baixo peso: <percentil 5.

- Risco de sobrepeso: $\geq$ percentil $85<$ percentil 95.

- Normalidade: entre o percentil 5 e $<$ percentil 85.

Para a categoria "Sobrepeso" utilizou-se como critério $\geq$ percentil 95, ponto de corte proposto por um grupo de estudiosos do Clinical Guidelines for Overweight in Adol escent Preventive Services, Estados Unidos (Himes \& Dietz, 1994)

A tomada do peso e da altura foi realizada utilizando-se os procedimentos recomendados pela OMS (WHO, 1995).

Os dados foram descritos por meio de médias, medianas, desvios padrão e proporções.

Utilizou-se o teste do Qui-Quadrado para verificar as relações entreas variáveis sexo e estado nutricional, sexo e idade.

\section{Resultados}

Dos 125 alu nos que foram selecionados para compor a amostra, foram obtidos dados de 92 alunos (74,2\%). A perda de $25,8 \%$ deveu-se a alguns fatores tais como: a recusa dos próprios alunos selecionados, a não-permissão dos pais ou responsáveis e a não-devolução da Carta de Consentimento.

Em relação à renda familiar, $28,3 \%$ dos adolescentes encontraram-se na categoria entre 10 e 20 salários mínimos. Todavia, 23,9\% dos entrevistados não forneceram a informação sobre renda. A média da renda foi de $\mathrm{R} \$ 2.260,00$ (DP $=\mathrm{R} \$ 1.758,00)$

Para a variável escolaridade, tanto para os pais quanto para as mães, a mai or proporção foi encontrada na categoria 1o ao 3o colegial, sendo $28,3 \%$ e $30,4 \%$, respectivamente.

Com relação ao tipo de ocupação dos pais, observou-se que $21,7 \%$ encontravam-se na categoria "técnica científica, artística e assemeIhadas"; entretanto, $17,4 \%$ dos entrevistados não forneceram a informação. No caso das mães, 21,7\% encontravam-se nessa categoria e 22,8\% pertencem à categoria "administrativa"; somente 9,8\% não informaram.

Em relação à faixa etária, verificou-se que $53,5 \%$ dos adolescentes do sexo masculino estavam na faixa dos 13-14 anos; para o sexo feminino, 44,9\% estavam na faixa de $11-12$ anos e 44,9\% entre 13- 14 anos. A média de idade foi de 12,96 anos (DP $=1,24$ anos).

A Tabela 1 descreve o estado nutricional dos adol escentes por sexo. Para o sexo masculino, 58,1\% foram considerados normais. Porém, $27,9 \%$ e 4,7\% foram classificados com risco de sobrepeso e sobrepeso, respectivamente, totalizando 32,6\% de adolescentes. As adolescentes apresentaram melhor situação nutricional, uma vez que $71,5 \%$ foram classificadas como normais, mas deve-se ressaltar que $16,3 \%$ foram consideradas com sobrepeso e 10,2\% com risco de sobrepeso, totalizando $26,5 \%$. Pode ser observado que apenas 5,5\% dos adolescentes entrevistados (9,3\% para o sexo masculino e 2,0\% para o sexo feminino) apresentaram-se com baixo peso. Houve diferença estatisticamente significativa entre os sexos quanto ao estado nutricional $(p<0,05)$.

A Tabela 2 mostra a distribuição dos adolescentes conforme a faixa etária e estado nutricional. Para a faixa de $11-13$ anos, $62,7 \%$ foram considerados normais e 32,8\% com Sobrepeso e Risco de Sobrepeso. Para a faixa etária de 1417 anos, 72,0\% foram considerados normais, $28,0 \%$ com sobrepeso e risco de sobrepeso. Não houve diferença estatisticamente significativa quanto ao estado nutricional nas diferentes faixas etárias $(p>0,05)$.

\section{Discussão}

Avaliando o estado nutricional dos adolescentes deste estudo, a maior porcentagem de ambos os sexos foi classificada como normal, e o I MC médio encontrado para o sexo masculino foi de 20,11, estando entre os percentis 50 e 85 , 
Distribuição dos adolescentes segundo sexo e estado nutricional, 1998.

\begin{tabular}{|c|c|c|c|c|c|c|}
\hline \multirow[t]{3}{*}{ Estado nutricional* } & \multicolumn{4}{|c|}{ Sexo } & \multicolumn{2}{|c|}{ Total } \\
\hline & \multicolumn{2}{|c|}{ Masculino } & \multicolumn{2}{|c|}{ Feminino } & & \\
\hline & $\mathrm{n}$ & $\%$ & $n$ & $\%$ & $n$ & $\%$ \\
\hline Normal & 25 & 58,1 & 35 & 71,5 & 60 & 65,2 \\
\hline Sobrepeso & 2 & 4,7 & 8 & 16,3 & 10 & 10,9 \\
\hline Risco sobrepeso & 12 & 27,9 & 5 & 10,2 & 17 & 18,5 \\
\hline Baixo peso & 4 & 9,3 & 1 & 2,0 & 5 & 5,5 \\
\hline Total & 43 & 100,0 & 49 & 100,0 & 92 & 100,0 \\
\hline
\end{tabular}

* classificação de acordo com o valor do percentil do Índice de Massa Corporal (IMC).

$\chi^{2}=9,60 ; p=0,022$

Tabela 2

Distribuição dos adolescentes segundo idade e estado nutricional, 1998.

\begin{tabular}{|c|c|c|c|c|c|c|c|c|c|c|}
\hline \multirow[t]{2}{*}{ Idade (anos) } & \multicolumn{2}{|c|}{ Normal } & \multicolumn{2}{|c|}{ Sobrepeso } & \multicolumn{2}{|c|}{$\begin{array}{l}\text { Estado nutricional } \\
\text { Risco de } \\
\text { sobrepeso }\end{array}$} & \multicolumn{2}{|c|}{ Baixo peso } & \multicolumn{2}{|c|}{ Total } \\
\hline & $n$ & $\%$ & $n$ & $\%$ & $n$ & $\%$ & $n$ & $\%$ & $n$ & $\%$ \\
\hline $11-13$ & 42 & 62,7 & 10 & 14,9 & 12 & 17,9 & 3 & 4,5 & 67 & 100,0 \\
\hline $14-17$ & 18 & 72,0 & 0 & - & 5 & 20,0 & 2 & 8,0 & 25 & 100,0 \\
\hline Total & 60 & 65,2 & 10 & 10,9 & 17 & 18,5 & 5 & 5,4 & 92 & 100,0 \\
\hline
\end{tabular}

$\chi^{2}=4,43 ; p=0,218$

e para o sexo feminino, o IMC médio foi de 21,04 , correspondendo aos mesmos percentis anteriores.

Algumas pesquisas real izadas em países em desenvolvimento como Índia, Nigéria e China (Brabin et al., 1997; Chatuverdi et al., 1996; Wang et al., 1998) e em desenvolvidos como a Irlanda (Hurson \& Corish, 1997) mostraram prevalências de sobrepeso inferiores tanto comparadas com as pesquisas anteriormente citadas como com o presente estudo. No entanto, nem todos esses estudos utilizaram os mesmos critérios de classificação que o nosso.

O valor médio do IMC encontrado no presente estudo, para o sexo masculino $(20,11 \pm$ 3,11 ) e para o sexo feminino $(21,04 \pm 3,73)$, mostrou-se superior ao encontrado em estudo mexicano conduzido por Tena-Flores \& Frisancho (1997).

Segundo Monteiro (1998a), a tendência secular da obesidade na população de crianças e adolescentes americanos pode ser observada valendo-se de inquéritos realizados entre os anos 60 e 90 . Entre os anos 60 e 70, observamse discretos aumentos na freqüência da obesi- dade em ambos os sexos. Entre 1980 e 1994, foram observados aumentos acentuados na prevalência da obesidade em crianças e adolescentes de todas as faixas etárias e em ambos os sexos.

Foi demonstrado que $40 \%$ das crianças que são obesas até os sete anos tornam-se adultos obesos, enquanto $70-80 \%$ de adolescentes obesos tornam-se adultos obesos além de possuírem maiores riscos de morbi-mortalidade (Kolata, 1986, apud Ortega et al., 1995). Hoffmans et al. (1989) verificaram em um estudo de caso, em adolescentes do sexo masculino, que o risco de mortalidade por doenças cardiovasculares foi maior para o grupo que apresentava IMC $\geq 25$.

Must et al. (1992) observaram, em um estudo com adolescentes de ambos os sexos, que para o sexo masculino, o sobrepeso foi associado com um risco maior de doenças cardiovasculares e câncer de cólon e reto.

A prevalência de sobrepeso encontrada neste estudo é semelhante àquelas encontradas em grande parte das pesquisas realizadas no Brasil (Fonseca et al., 1998; Nuzzo, 1998; Si- 
chieri et al., 1998), em países desenvolvidos e em outros países em desenvolvimento (Anta et al., 1996; Brugman et al., 1997; Ortega et al., 1995), demonstrando que o sobrepeso em adolescentes é um problema crescente e preocupante, havendo a necessidade de estudo em nível nacional, neste grupo etário, para verificar a dimensão do problema e criar estratégias de prevenção e controle.

\section{Referências}

ANTA, R. M. O.; CARVAJALES, P. A.; REQUEJO, A. M . M.; SOBALER, A. M. L.; SOBRADO, M. R. R. \& GONZALEZ-FERNANDEZ, M., 1996. Hábitos alimentarios e ingesta de energía y nutrientes en adolescentes con sobrepeso en comparación con los de peso normal. Anales Españoles de Pediatría, 44:203-208.

BRABIN, L.; IKIMALO, J .; DOLLIMORE, N.; KEMP, J .; IKOKWU-WONODI, C. \& BABATUNDE, S., 1997. How do they grow? A study of south-eastern Nigerian adolescent girls. Acta Paediatrica, 86: 1114-1120.

BRUGMAN, E.; MEULMEESTER, J. F.; SPEE-VAN DER WEKLE, A.; BEUKER, R. J.; ZAADSTRA, B. M. \& RADDER, J. J., 1997. Dieting, weight and health in adolescents in the Netherlands. International Journal of Obesity, 21:54-60.

CHATUVERDI, S.; KAPIL, U.; GNANASEKARAN, N.; SACHDEV, H. P. S.; PANDEY, R. M. \& BHANTI, T., 1996. Nutrient intake amongst adolescent girls belonging to poor socioeconomic group of rural area of Rajasthan. Indian Pediatric, 33:197-201.

DANIELS, S. R.; KHOURY, P. R. \& MORRISON, J. A., 1997. The utility of body mass index as a measure of body fatness in children and adolescents: Differences by race and gender. Pediatrics, 99: 804807.

DIETZ, W. H., 1998. Health consequences of obesity in youth: Childhood predictors of adult disease. Pediatrics, 101(Sup.):518-525.

FONSECA, V. M.; SICHIERI, R. \& VEIGA, G. V., 1998. Fatores associados à obesidade em adolescentes. Revista deSaúde Pública, 32:541-549.

HEALTH, G. W.; PRATT, M.; WARREN, C. W. \& KANN, L., 1994. Physical activity patterns in american high school students. Archives of Pediatrics and Adolescent Medicine, 148:1131-1136.

HILL, J. O. \& TROWBRIDGE, F. L., 1998. Childhood obesity: future directions and research priorities. Pediatrics, 101(Sup. 3):570-574.

HIMES, J. H. \& DIETIZ, W. H., 1994. Guidelines for overweight in adolescent preventive services: Recommen dations from an expert committee. American Journal of Clinical Nutrition, 59: 307-316.
HURSON, M. \& CORISH, C., 1997. Evaluation of lifestyle, food consumption and nutrient intake patterns among I rish teenagers. Irish Journal of Medical Science, 166:225-230.

HOFFMANS, M. D. A. F.; KROM HOUT, D. \& COULANDER, C. L., 1989. Body mass index at the age of 18 and its effects on 32-year-mortality from coronary heart disease and cancer. Journal of Clinical Epidemiology, 42:513-520.

IBGE (Fundação Instituto Brasileiro de Geografia e Estatística), 1990. Pesquisa Nacional por Amostra de Domicílio . Anexo 1 - Grupos Ocupacionais e Ocupações. v. 1443. Rio de Janeiro: IBGE.

IBGE (Fundação Instituto Brasileiro de Geografia e Estatística), 1997. Anuário Estatístico do Brasil. Rio de Janeiro: IBGE.

KENNEDY, E. \& GOLDBERG, J., 1995. What are american children eating? Implications for public policy. Nutrition Reviews, 53:111-126.

MONTEIRO, C. A., 1998a. Epidemiologia da obesidade. In: Obesidade (A. Halpern, ed.), pp. 15-30, São Paulo: Editora Lemos Editorial.

MONTEIRO, J. C., 1998b. Obesidade: Diagnóstico, métodos e fundamentos. In: Obesi dade (A. Halpern, ed.), pp. 31-53, São Paulo: Editora Lemos Editorial.

MS (Ministério da Saúde), 1990. Pesquisa Nacional sobre Saúdee Nutrição: Perfil de Crescimento da População Brasileira de 0 a 25 Anos. Brasília: MS.

MUST, A.; JACQUES, P. F.; DALLAL, G. E.; BAJEMA, C. J. \& DIETZ, W. H., 1992. Long-term morbidity and mortality of overweight adolescents: A follow-up of the Harvard Growth Study of 1922 to 1935. New England Journal of Medicine, 5:1350-1355.

NUZZO, L., 1998. Avaliação do Estado Nutricional de Adol escentes de uma Instituição Particular deEnsino. Dissertação de Mestrado, São Paulo: Faculdade de Saúde Pública, Universidade de São Paulo.

OMS (Organización Mundial de la Salud), 1995. La Salud de Ios Jóvenes: Un Reto y una Esperanza. Geneva: OMS.

OPS (Organización Panamericana de la Salud),1995. La Salud de los Adol escentes y los Jóvenes en Ias 
Américas: Escribiendo el Futuro. Comunicación para la Salud 6. Washington, DC: OPS.

ORTEGA, R. M .; REQUEJO, A. M.; ANDRES, P.; LOPEZSOBALER, A. M.; REDONDO, R. \& GONZALEZFERNANDEZ, M., 1995. Relationship between diet composition and body mass index in a group of Spanish adolescents. British Journal of Nutrition, 74:765-773.

PIETROBELLI, A.; FAITH, M. S.; ALLISON, D. B.; GALLAHER, D., CHIUMELLO, G. \& HEYMSFIELD, C., 1998. Body mass index as a measure of adiposity among children and adolescents: A validation study. Journal of Pediatric, 132:204-210.

ROSENBAUM, M. \& LEIBEL, R. L., 1998. The physiology of body weight regulation: Relevance to the etiology of obesity in children. Pediatrics, 101 (Sup. 3): 525-539.

SAITO, I. M., 1993. Nutrição. In: Medicina do Adolescente( V. Coates, org.), pp. 37-50, São Paulo: Editora Sarvier.
SICHIERI, R., 1998. Epidemi ologia da Obesidade. Rio deJaneiro: Editora UERJ.

SILVA, N. N., 1998. Amostragem Probabilística. São Paulo: Edusp.

TENA-FLORES, J. A. \& FRISANCHO, A. R., 1997. Crecimiento antropométrico de la población en zonas rurales y suburbanas de Durango, México. Archivos Latinoamericanos deNutrición, 47:105-109.

TROIANO, R. P. \& FLEGAL, K. M., 1998. Overweight children and adolescents: Description, epidemiology and demographics. Pediatrics, 101(Sup. 3): 497-504.

WANG, Y.; POPKIN, B. \& ZHAI, F., 1998. The nutritional status and dietary pattern of Chinese adolescents, 1991 and 1993. European Journal of Clinical Nutrition, 52:908-916.

WHO (World Health Organization), 1995. Physical Status: The Use and Interpretation of Anthropometry. Technical Report Series 854. Geneva: WHO. 